\title{
Diagnosis of transition zone prostate cancer by multiparametric MRI: added value of MR spectroscopic imaging with sLASER volume selection
}

\author{
Neda Gholizadeh ${ }^{1}$, Peter B. Greer ${ }^{2,3}$, John Simpsonn ${ }^{2,3}$, Jonathan Goodwin ${ }^{2,3}$, Caixia Fu' ${ }^{4}$, Peter Lau ${ }^{5,6}$, \\ Saabir Siddique ${ }^{5,6}$, Arend Heerschap ${ }^{7}$ and Saadallah Ramadan ${ }^{1,6^{*}}$ (i)
}

\begin{abstract}
Background: Current multiparametric MRI (mp-MRI) in routine clinical practice has poor-to-moderate diagnostic performance for transition zone prostate cancer. The aim of this study was to evaluate the potential diagnostic performance of novel ${ }^{1} \mathrm{H}$ magnetic resonance spectroscopic imaging (MRSI) using a semi-localized adiabatic selective refocusing (sLASER) sequence with gradient offset independent adiabaticity (GOIA) pulses in addition to the routine $\mathrm{mp}-\mathrm{MRI}$, including T2-weighted imaging (T2WI), diffusion-weighted imaging (DWI) and quantitative dynamic contrast enhancement (DCE) for transition zone prostate cancer detection, localization and grading.

Methods: Forty-one transition zone prostate cancer patients underwent mp-MRI with an external phased-array coil. Normal and cancer regions were delineated by two radiologists and divided into low-risk, intermediate-risk, and high-risk categories based on TRUS guided biopsy results. Support vector machine models were built using different clinically applicable combinations of T2WI, DWI, DCE, and MRSI. The diagnostic performance of each model in cancer detection was evaluated using the area under curve (AUC) of the receiver operating characteristic diagram. Then accuracy, sensitivity and specificity of each model were calculated. Furthermore, the correlation of mp-MRI parameters with low-risk, intermediate-risk and high-risk cancers were calculated using the Spearman correlation coefficient. Results: The addition of MRSI to T2WI + DWI and T2WI + DWI + DCE improved the accuracy, sensitivity and specificity for cancer detection. The best performance was achieved with T2WI + DWI + MRSI where the addition of MRSI improved the AUC, accuracy, sensitivity and specificity from 0.86 to $0.99,0.83$ to $0.96,0.80$ to 0.95 , and 0.85 to 0.97 respectively. The (choline + spermine + creatine)/citrate ratio of MRSI showed the highest correlation with cancer risk groups $(r=0.64, p<0.01)$.
\end{abstract}

Conclusion: The inclusion of GOIA-sLASER MRSI into conventional mp-MRI significantly improves the diagnostic accuracy of the detection and aggressiveness assessment of transition zone prostate cancer.

Keywords: Prostate cancer, Multiparametric MRI, ${ }^{1} \mathrm{H}$ MR spectroscopic imaging, GOIA-SLASER, SVM

*Correspondence: saadallah.ramadan@newcastle.edu.au

${ }^{6}$ Hunter Medical Research Institute (HMRI) Imaging Centre, New Lambton Heights, NSW, Australia

Full list of author information is available at the end of the article

\section{Background}

Prostate cancer $(\mathrm{PCa})$ is the most common malignancy and the fourth leading cause of cancer death among males worldwide [1]. Almost $30 \%$ of PCa lesions occur in the transition zone (TZ) [2]. TZ cancer has a relatively original author(s) and the source, provide a link to the Creative Commons licence, and indicate if changes were made. The images or other third party material in this article are included in the article's Creative Commons licence, unless indicated otherwise in a credit line to the material. If material is not included in the article's Creative Commons licence and your intended use is not permitted by statutory regulation or exceeds the permitted use, you will need to obtain permission directly from the copyright holder. To view a copy of this licence, visit http://creativecommons.org/licenses/by/4.0/. The Creative Commons Public Domain Dedication waiver (http://creativeco mmons.org/publicdomain/zero/1.0/) applies to the data made available in this article, unless otherwise stated in a credit line to the data. 
low Gleason score (GS), local stage and biochemical recurrence rate in comparison with peripheral zone (PZ) $\mathrm{PCa}$ [3]. Due to difficulties in localizing TZ by digital rectal exam, sampling of $\mathrm{TZ}$ cancer by routine transrectal ultrasound (TRUS)-guided biopsy and low specificity of prostate specific antigen (PSA) as cancer marker, there is a high potential risk for missing TZ lesions [4]. Magnetic resonance imaging (MRI) with its non-invasive soft tissue contrast capability has the potential to overcome these limitations for the detection of TZ cancer [5]. T2-weighted imaging (T2WI) provides the best anatomical images of TZ and PZ. However, TZ often contains benign prostatic hyperplasia (BPH), which may have low-signal intensity on T2WI similar to that of cancer [6]. Furthermore, the clinical application of multiparametric MRI (mp-MRI) including T2WI, diffusion-weighted imaging (DWI) and dynamic contrast enhanced (DCE) imaging as defined by the Prostate Imaging Reporting and Data System (PI-RADS V2) has shown moderate diagnostic performance for TZ cancer [7]. The overlap of apparent diffusion coefficient (ADC) values between cancer and $\mathrm{BPH}$ in $\mathrm{TZ}$ is the main limitation of DWI [8]. DCE has a minor role in TZ cancer detection because $\mathrm{BPH}$ in $\mathrm{TZ}$ can be highly vascularized and shows rapid and high level enhancement similar to $\mathrm{PCa}$ [9]. The diagnostic potential of proton MR spectroscopic imaging (MRSI) in combination with anatomical and functional MRI for the improvement of PCa detection, localization and characterization has already been demonstrated [10, 11]. Point-resolved spectroscopy (PRESS) is the most commonly used pulse sequence for prostate MRSI mainly because of its commercial availability, but it suffers from chemical shift displacement error, long acquisition time and bad slice profiles, which causes unpredictable lipid signal contamination. These problems were addressed by using a semi-localized adiabatic selective refocusing pulse sequence (sLASER) with gradient-modulated offset-independent adiabatic (GOIA) pulses (GOIAsLASER), which resulted in much cleaner MR spectra of the prostate [12]. To our knowledge, there have been no reports on the diagnostic performance of MRSI GOIAsLASER within routine clinical prostate mp-MRI exams.

The purpose of this study was to evaluate the diagnostic performance of individual and combined mp-MRI parameters, employed for PI-RADS evaluations, and GOIA-sLASER MRSI using an external phased-array coil for $\mathrm{TZ}$ cancer detection, localization and grading.

\section{Methods}

\section{Patients}

In total, 45 patients with biopsy-proven $\mathrm{TZ}$ cancer and one biopsy-negative subject were consecutively enrolled in this study. Four patients were excluded from the study due to poor-quality data. The study protocol was approved by the local Human Research Ethics Committee and each patient signed a consent form before the MRI examination. Depending on prostate gland size, at least sixteen TRUS guided biopsy cores were obtained from each patient between six to eight weeks before the MRI examination.

\section{MRI acquisition protocol}

Prostate mp-MRI was performed on a whole-body $3 \mathrm{~T}$ MR scanner using an eighteen-channel phased-array coil (MAGNETOM Prisma, Siemens Healthcare, Erlangen, Germany) and without endorectal coil. The mpMRI protocol included axial, coronal and sagittal T2WI, DWI and three-dimensional (3D) MRSI. The last step in this protocol was DCE MRI for which paramagnetic gadolinium (Gadovist ${ }^{\circledR}$, Bayer Heathcare Pharmaceuticals, Berlin, Germany) was administered as bolus injection of $0.1 \mathrm{mmol} / \mathrm{kg}$ body weight with a power injector at $2.5 \mathrm{~mL} / \mathrm{s}$ and followed by a $15-\mathrm{mL}$ saline flush. A DCE time series was acquired with a T1-weighted sequence including 4 baseline acquisitions before the injection. Details of the mp-MRI acquisition parameters are summarized in Table 1.

\section{MRI prostate mapping and pre-processing}

Bias correction, noise reduction and intensity standardisation were applied on T2WI [13].

ADC maps were calculated inline from DWI's using a mono-exponential fitting of b-values $0,400,800$ and $1600 \mathrm{~s} / \mathrm{mm}^{2}$ in syngo software.

DCE-MR images were processed by a Siemens dedicated Tissue4D module for dynamic analysis of DCE MR with an implementation of the Tofts model and an assumed arterial input function (AIF) [14]. After motion correction, optimal AIFs were selected from slow, intermediate and fast population-averaged options, considering individual volume of interest (VOI) curves. The quantitative variables derived from T1 maps were the volume transfer constant $\left(\mathrm{K}^{\text {trans }}, \mathrm{min}^{-1}\right)$, the rate constant $\left(\mathrm{K}_{\mathrm{ep}}, \mathrm{min}^{-1}\right)$ and the area under gadolinium curve (iAUGC, $\mathrm{mmol} \mathrm{min} / \mathrm{L}$ ).

Citrate (Cit), creatine (Cr), choline (Cho) and spermine $(\mathrm{Spm})$ tissue concentrations within a voxel were determined by the automated peak fitting algorithm LCModel (Version 6.3-1L) using metabolite basis sets. Two Cit ratios, $((\mathrm{Cho}+\mathrm{Spm}+\mathrm{Cr}) / \mathrm{Cit}$ and $\mathrm{Cho} / \mathrm{Cit})$, and the $\mathrm{Cho} / \mathrm{Cr}$ ratio were calculated for each voxel within a selected region (vide infra).

\section{Tissue segmentation}

All patients underwent at least 16-core (S16C) transrectal and transperineal ultrasound-guided biopsy using a 
Table $1 \mathrm{MRI}$ acquisition parameters for prostate multiparametric MRI (mp-MRI)

\begin{tabular}{|c|c|c|c|c|c|c|}
\hline & T2WI (Axial) & T2WI (Coronal) & T2WI (Sagittal) & DWI & DCE & MRSI \\
\hline Sequence & 2DTSE & 2DTSE & 2DTSE & EPI & 3D VIBE & GOIA-SLASER \\
\hline $\mathrm{TR}(\mathrm{ms})$ & 4300 & 3620 & 4500 & 3400 & 4.88 & 820 \\
\hline TE (ms) & 102 & 104 & 90 & 53 & 1.77 & 88 \\
\hline Averages & 2 & 3 & 3 & 16 & 1 & 4 \\
\hline $\begin{array}{l}\text { Flip angle } \\
\text { (degree) }\end{array}$ & 160 & 160 & 128 & 90 & 15 & $90-4 \times 180$ \\
\hline Thickness (mm) & 3 & 3 & 3 & 3 & 3 & 7 \\
\hline Gap (mm) & 0 & 0 & 0 & 0 & 0 & 0 \\
\hline FOV $(\mathrm{mm})$ & $200 \times 200$ & $200 \times 200$ & $240 \times 240$ & $256 \times 256$ & $260 \times 260$ & $84 \times 84 \times 70$ \\
\hline b-value $\left(\mathrm{s} / \mathrm{mm}^{2}\right)$ & NA & $\mathrm{NA}$ & NA & $0,400,800$ and 1600 & NA & NA \\
\hline Temporal resolution (s) & NA & NA & NA & NA & 4.8 & NA \\
\hline Matrix & $448 \times 448 \times 30$ & $448 \times 448 \times 30$ & $320 \times 320 \times 24$ & $84 \times 128 \times 24$ & $192 \times 192 \times 24$ & $12 \times 12 \times 10$ \\
\hline Spatial resolution $\left(\mathrm{mm}^{3}\right)$ & $0.45 \times 0.45 \times 3$ & $0.45 \times 0.45 \times 3$ & $0.75 \times 0.75 \times 3$ & $2 \times 2 \times 3$ & $1.35 \times 1.35 \times 3$ & $7 \times 7 \times 7$ \\
\hline Acquisition time (min) & 3.19 & 4.51 & 4.05 & 6.26 & 6.23 & 8.42 \\
\hline
\end{tabular}

TR repetition time, TE echo time, FOV field of view, TSE turbo spin echo, EPI echo planar imaging, VIBE volumetric interpolated breath-hold examination, GOIA-sLASER gradient offset independent adiabaticity with semi-localized adiabatic selective refocusing, NA not available/not applicable

reusable biopsy gun. Two independent radiologists, one with more than twenty years' experience (P.L.) and one with thirteen years' experience (S.S.) in prostate radiology, evaluated all MRI images in conjunction with the biopsy reported cancer locations. Radiologists visually matched ADC maps, baseline images (b-value $=0 \mathrm{~s} /$ $\mathrm{mm}^{2}$ ), and corresponding T2WI slice locations and gland anatomy (apex, mid-gland area and base).

In total, 61 cancer regions with biopsy-proven positive TZ cancer (1-3 cancer regions per patient) were manually delineated on T2WI by the two radiologists and these were subsequently co-registered to the corresponding ADC, DCE and MRSI maps. To maximize the identification accuracy of the cancer regions, only concurrent reporting of cancer ROIs was used. Then, the two radiologists in consensus selected 73 normal regions from $\mathrm{TZ}$ with negative biopsies (1-2 normal regions per patient). Each cancer or normal region was selected over one ROI on a single slice or multiple ROIs on multiple slices for each patient. The ROIs on multiple slices for each tissue were summarised per mean value to eliminate bias.

\section{Assessment of histologic tumor grade}

Cancerous tissues were sub-divided according to GS into three main risk groups to evaluate the value of mp-MRI parameters to discriminate between different cancer grades. These risk groups are (a) 37 regions with GS of $3+3$ as low-risk, (b) 9 regions with GS of $3+4$ as intermediate-risk and (c) 15 regions with GS $\geq 4+3$ as highrisk. Each cancer region parameter on mp-MRI maps was subsequently correlated to the matching GS group based on biopsy results.

\section{Classification and statistical analysis}

A non-parametric Mann-Whitney U-test with Bonferroni correction was performed for each pair group to determine significant difference between the mean values of normal and cancer regions of mp-MRI parameters.

We developed a machine learning platform for $\mathrm{mp}$ MRI including support vector machine (SVM) classifications with a radial basis function kernel (RBF-SVM) and area under receiver operator characteristic (ROC) analyses using an in-house Matlab routine to evaluate the diagnostic performance of models with different parametric combinations: T2WI + DWI, T2WI+DWI+DCE, $\mathrm{T} 2 \mathrm{WI}+\mathrm{DWI}+\mathrm{MRSI}$, and T2WI + DWI + DCE + MRSI. Mp-MRI parameters involved were $\mathrm{ADC}, \mathrm{K}^{\text {trans }}, \mathrm{K}_{\mathrm{ep}}$, iAUGC, $(\mathrm{Cho}+\mathrm{Spm}+\mathrm{Cr}) / \mathrm{Cit}, \mathrm{Cho} / \mathrm{Cit}$ and $\mathrm{Cho} / \mathrm{Cr}$. Models were developed with mp-MRI parameters with a statistically significant difference between each pair group. The sensitivity, specificity and overall accuracy of each classifier are reported.

The correlation between risk groups (low-risk, intermediate-risk and high-risk) and mp-MRI parameters was measured with Spearman correlation coefficient (r) (IBM SPSS). Then, mp-MRI parameters with $|r| \geq 0.25$ were used to develop RBF-SVM models between cancer risk groups. These models were used to evaluate the diagnostic performance of mp-MRI parameters to differentiate cancer risk groups. All evaluations were based on biopsy results. 
Optimal kernel parameters for RBF-SVM were calculated by a grid search approach [15]. The performance of the SVM classification models was determined by leaveone-out cross-validation.

McNemar tests were used for pairwise comparisons of sensitivity and specificity between different mp-MRI models, while Delong test was used to compare the area under curves (AUCs). A $p$ value $<0.05$ was considered statistically significant.

\section{Results}

\section{Multiparametric MRI of prostate cancer patients}

To avoid inclusion of low-quality data, the MR data from four patients were excluded from the study as they showed distortion artefacts on DWI and had a $\mathrm{FWHM}_{\text {water }}>50 \mathrm{~Hz}$ for the whole prostate gland, related to patients' movements and motion during scanning. Clinical and demographic data of the remaining 41 patients are summarized in Table 2.

Employing a phased-array receive coil (without an endorectal coil), the application of GOIA-sLASER MRSI to the prostate produced MR spectra showing wellresolved metabolite signals with adequate SNR (Figs. 1G, $2 \mathrm{G}$ and $3 \mathrm{G})$.

Figure 1 shows MR data of a prostate which was biopsy-proven negative for $\mathrm{PCa}$. The negative-biopsy areas in the left and right $\mathrm{TZ}$ appeared as low-signal intensity on T2WI and the ADC map (restricted diffusion) and as high-signal intensity on high b-value DWI, $\mathrm{K}^{\text {trans }}$ and iAUGC maps (Fig. 1A-D, F). However, these areas appeared as normal on $\mathrm{K}_{\mathrm{ep}}$ maps (Fig. 1E). Moreover, the MR spectra, derived from the MRSI exam, of these (biopsy cancer negative) ROIs exhibited high Cit and low Cho signals (Fig. 1G) representing normal prostate tissue and thus providing vital information indicative of the absence of cancer tissue.
Figure 2 shows data of an mp-MRI exam obtained from a patient with a region in the prostate with low-risk cancer $(\mathrm{GS}=3+3)$ in the left $\mathrm{TZ}$ ( arrows). This region appeared as low-signal intensity on T2WI and on the ADC map (Fig. 2A, B) and high-signal intensity on high b-value DWI and on pharmacokinetic maps derived from DCE (Fig. 2C-F). The MR spectra obtained from this ROI exhibited relatively high Cho signals and low Cit signals (Fig. 2G, right). A negative biopsy area in the right TZ of the prostate of this patient (arrows) also appeared as lowsignal intensity on T2WI and the ADC map (restricted diffusion) and as high-signal intensity on high b-value DWI and pharmacokinetic maps (Fig. 2A-F), all suggesting the presence of cancer tissue. However, the MR spectra of this region, derived from the 3D MRSI exam, exhibited normal high Cit signals and low Cho signals (Fig. 2G, left), in complete agreement with the biopsy results that this region contains no cancer.

In patients with high-risk $\mathrm{TZ}$ prostate cancers, tumor tissue presented as low-signal intensity on T2WI and ADC maps and high-signal intensity on high b-value DWI and pharmacokinetic parameter maps of DCE exams (Fig. 3A-F). Normal TZ tissue showed higher signal intensity on ADC maps (Fig. 3B). However, quantitative DCE parameters maps generally showed inconclusive enhancements making them ineffective in the differentiation of central cancer tissue from normal tissue. MRSI spectral maps of the entire prostate showed low-signal intensity levels for Cit and high-intensity levels for Cho in tumor areas (Fig. 3G, left), whereas normal TZ tissue showed relatively high levels of Cit and low levels of Cho (Fig. 3G, right).

In addition, in five patients with both $\mathrm{TZ}$ and $\mathrm{PZ} \mathrm{PCa,}$ high quality MR spectra were obtained for PZ. Figure 4 is an example of a spectral map of the entire prostate gland of a man with biopsy-proven PZ and TZ PCa lesions.

Table 2 Demographic and clinical data of the prostate cancer patients enrolled in this study (A) and Gleason score of biopsies (B)

\begin{tabular}{llr}
\hline A) & Mean \pm SD & Range \\
\hline Age (years) & $66.31 \pm 7.19$ & $53-81$ \\
PSA $(\mathrm{ng} / \mathrm{mL})$ & $7.82 \pm 3.91$ & $2.5-18.9$ \\
Prostate volume $\left(\mathrm{cm}^{3}\right)$ & $45.01 \pm 17.23$ & $22.02-91.11$ \\
\hline B) Biopsy Gleason Score & Number of biopy-proven cancer tissue (total=61) & \% of total \\
\hline $3+3$ & 37 & 60.7 \\
$3+4$ & 9 & 14.7 \\
$4+3$ & 7 & 11.5 \\
$4+4$ & 6 & 9.8 \\
$4+5$ & 2 & 3.3 \\
\hline
\end{tabular}

PSA prostate specific antigen 

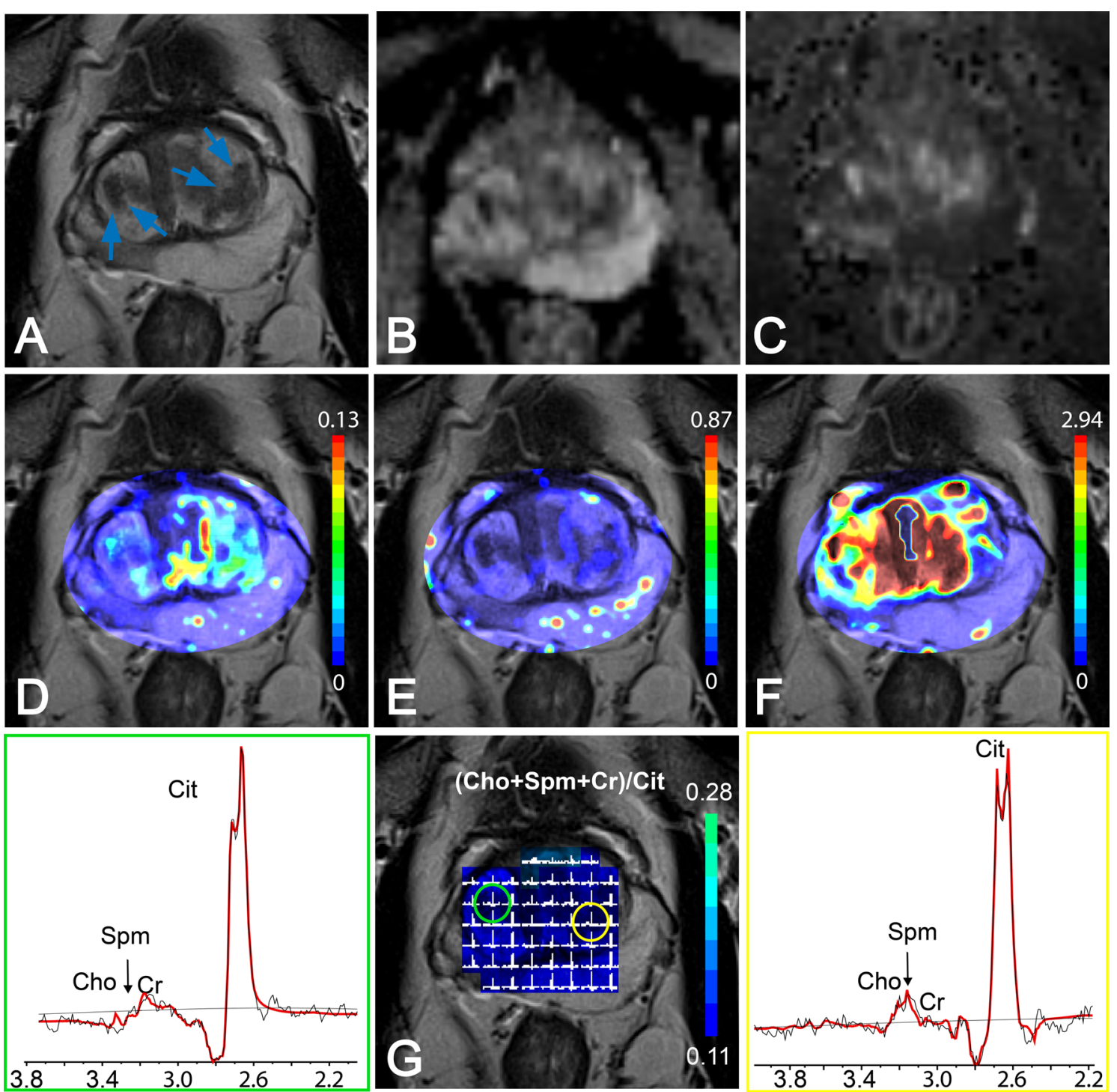

Fig. 1 Multiparametric MRI of the prostate of a 58-year-old man with an elevated serum PSA level of $6.8 \mathrm{ng} / \mathrm{mL}$ and cancer-negative biopsy results. A T2WI with low-signal intensity areas in the left and right transition zone (arrows) which were biopsy negative for cancer. B ADC map showing low-signal intensity for the same areas. High-signal intensity for these areas were seen on $\mathbf{C}$ high b-value DWI and on pharmacokinetic maps of D K ${ }^{\text {trans }}, \mathbf{E} K_{\text {ep }}$ and $\mathbf{F}$ iAUGC. G Middle: MRSI grid and color-coded map overlaid on T2WI. The spectra in the yellow box at the right side and in the green box at the left side represent voxels from histopathology confirmed normal tissue (circles)

The mean and standard deviation values of mp-MRI parameters in $\mathrm{TZ}$ cancer and normal tissue are given in Table 3. ADC values derived from DWI measurements were significantly lower in cancerous tissues than in normal tissues $(p<0.01)$. The pharmacokinetic parameters $\mathrm{K}^{\text {trans }}, \mathrm{K}_{\mathrm{ep}}$ and iAUGC, derived from DCE MRI, increased in cancer tissues compared to normal tissues $(p<0.05)$. The mean values of $(\mathrm{Cho}+\mathrm{Spm}+\mathrm{Cr}) / \mathrm{Cit}$, Cho/Cit and $\mathrm{Cho} / \mathrm{Cr}$ from the MRSI examinations in cancer ROIs were significantly higher than in normal tissues $(p<0.01)$. There was also a significant difference in all metabolite ratios of cancer compared to normal tissues in $\mathrm{PZ}$ $(p<0.01)$.

\section{Individual and combined MR imaging metrics to detect cancer tissue}

Figure 5 shows ROC curves of final RBF-SVM models for each pair of groups with and without MRSI (left side). These results demonstrated that MRSI significantly improved the AUC $(p<0.01)$ and also sensitivity and specificity $(p<0.01)$ in the detection of $\mathrm{TZ}$ cancers (bar charts on right side of Fig. 5). There was 

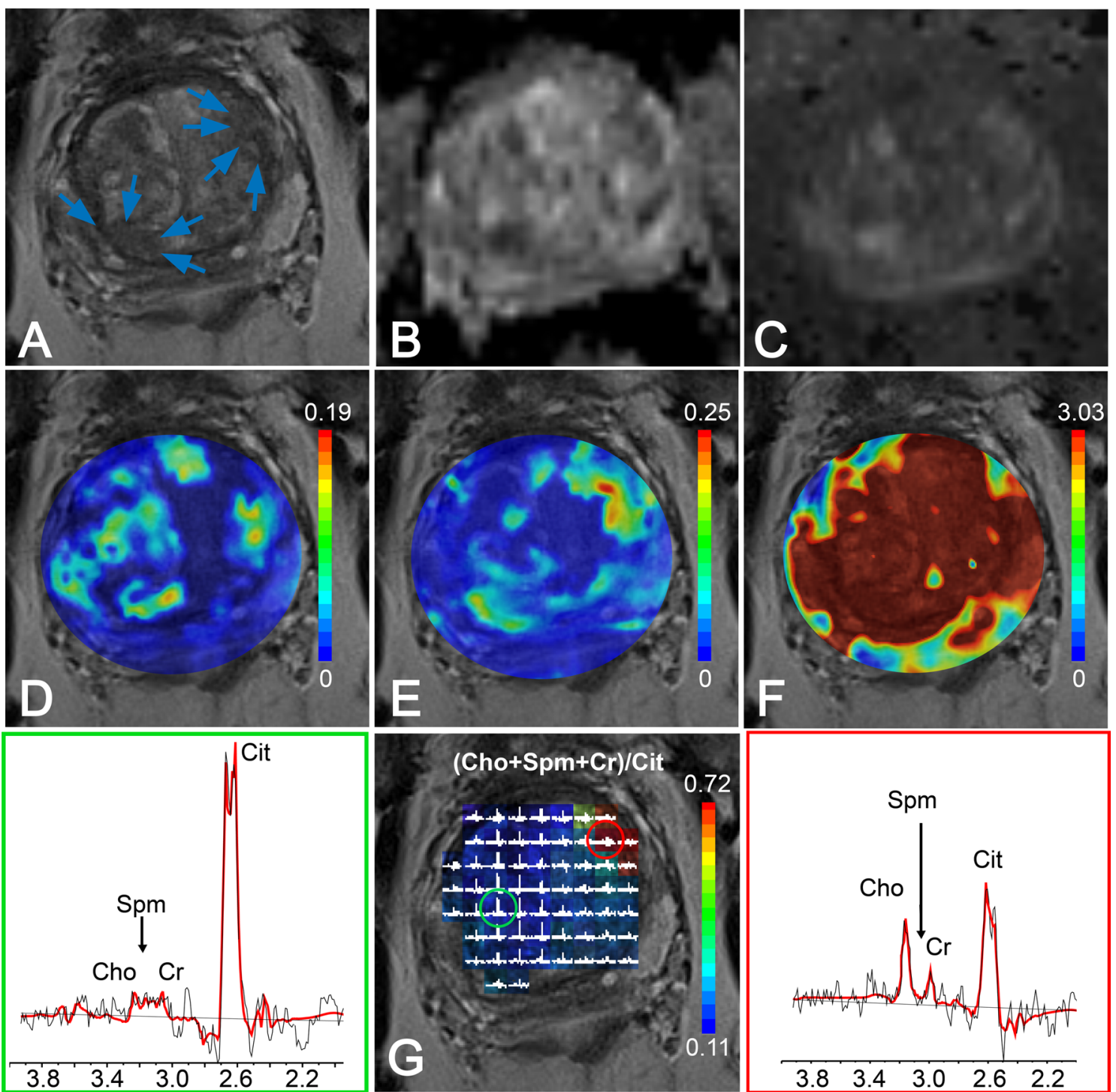

Fig. 2 Multiparametric MRI of the prostate of a 68-year-old man with a serum PSA level of $4.3 \mathrm{ng} / \mathrm{ml}$, who was diagnosed with a biopsy-proven Gleason $3+3$ cancer region in the left of the transition zone (arrows). The area on the right of the transition zone (arrows) had a cancer-negative biopsy outcome. A T2WI, B ADC map, C high b-value DWI, pharmacokinetic maps of calculated $\mathbf{D} \mathrm{K}^{\text {trans }}, \mathbf{E} \mathrm{K}_{\mathrm{ep}}$, F iAUGC and G MRSI grid and color-coded map overlaid on T2WI, with the red circle representing a voxel with cancer (spectrum in box at the left hand side) and the green circle representing a voxel with normal tissue (spectrum in box at the right hand side)

no significant difference between AUC, sensitivity and specificity of T2WI+DWI and T2WI+DWI+DCE $(p>0.05)$ and also between T2WI+DWI+MRSI and $\mathrm{T} 2 \mathrm{WI}+\mathrm{DWI}+\mathrm{DCE}+\mathrm{MRSI}$ models $(p>0.05)$.

For models without MRSI, T2WI + DWI achieved the highest AUC, accuracy and specificity at $0.86,0.83$ and 0.85 , respectively and the T2WI + DWI + DCE model yielded the highest sensitivity at 0.84 . The accuracy and specificity of the T2WI+DWI model was higher than that of the T2WI+DWI+DCE model.
For models including MRSI metabolite ratios that performed significantly better than without these ratios $(p<0.05)$, the T2WI+DWI+MRSI model achieved the highest AUC, accuracy, sensitivity and specificity at 0.99 , $0.96,0.95$ and 0.97 , respectively. There was no improvement in detection accuracy by adding DCE to the T2WI + DWI + MRSI model. The AUC, accuracy, sensitivity and specificity of all RBF-SVM models are summarized in Table 4. 

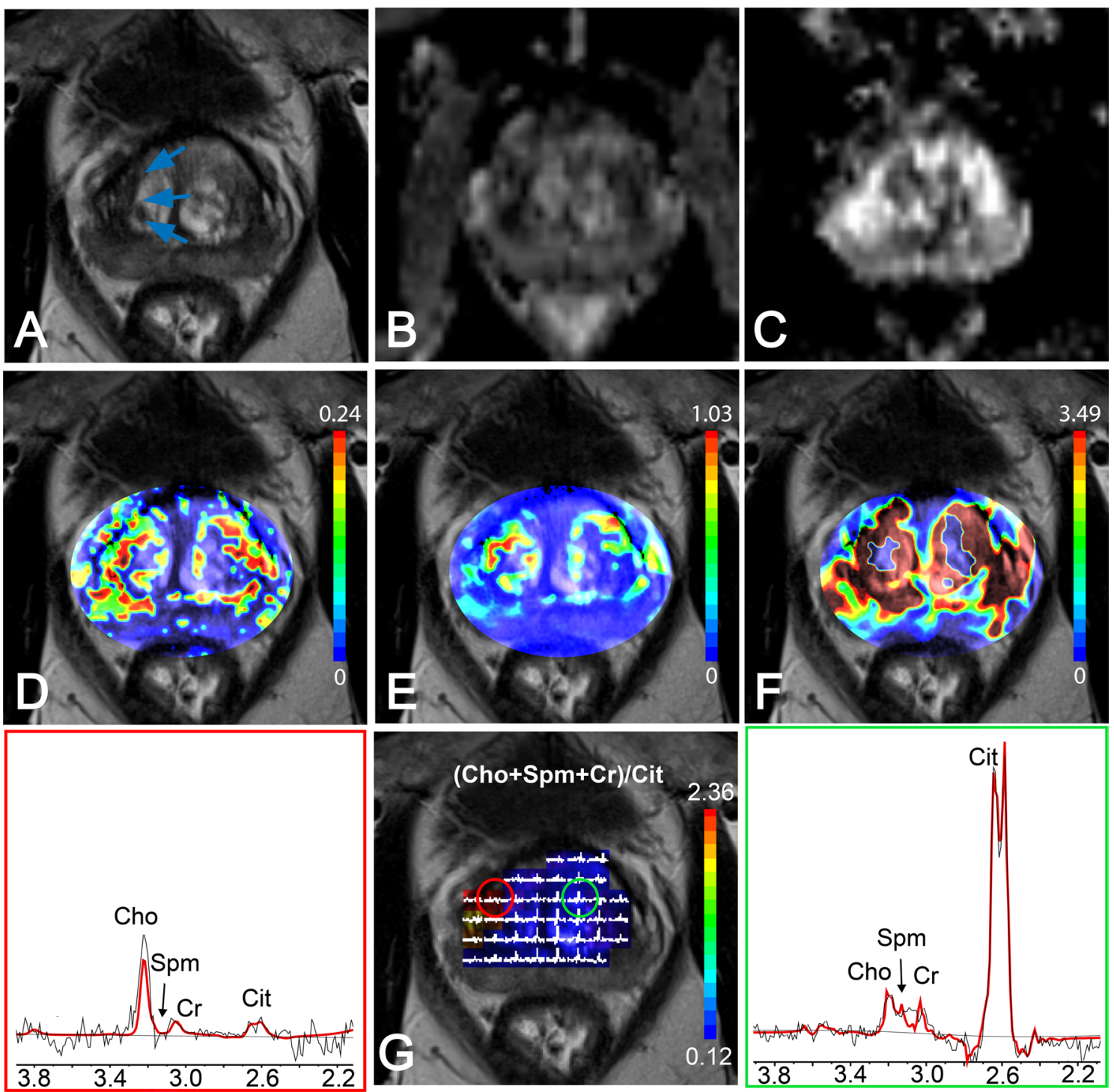

Fig. 3 Multiparametric MRI of the prostate of a 59-year-old patient with an elevated serum PSA level of $12.1 \mathrm{ng} / \mathrm{ml}$, who was diagnosed with a biopsy-proven prostate cancer with Gleason $4+4$ in the right side of the transition zone (arrows). A T2WI, B ADC map, C high b-value DWI, and pharmacokinetic maps of $\mathbf{D} \mathrm{K}^{\text {trans }}, \mathbf{E} \mathrm{K}_{\mathrm{ep}}$, and $\mathbf{F}$ iAUGC. G MRSI grid and color-coded map of (Cho $+\mathrm{Spm}+\mathrm{Cr}$ )/Cit values overlaid on T2WI, with red circle identifying a voxel in cancer region and green circle for a voxel in normal tissue. Left: MR spectrum from voxel of cancer tissue spectrum (red box). Right: MR spectrum from voxel of normal tissue (green box)

\section{Correlation of MR parameter values with tumor aggressiveness}

Correlation coefficients for the normalized T2WI, and the pharmacokinetic DCE parameters $\mathrm{K}_{\mathrm{ep}}$, and iAUGC with GS risk groups were very low and non-significant $(|\mathrm{r}|<0.25, p>0.05)$. A low, but significant, correlation was found for $\mathrm{K}^{\text {trans }}(\mathrm{r}=0.29, p<0.05)$. Compared to the DCE parameters, the average ADC demonstrated a better correlation with the different aggressiveness groups $(\mathrm{r}=-0.51, p<0.01)$. The $(\mathrm{Cho}+\mathrm{Spm}+\mathrm{Cr}) / \mathrm{Cit}$ derived from MRSI had the highest correlation with tumor aggressiveness $(\mathrm{r}=0.64, p<0.01)$. The correlation coefficients of $\mathrm{Cho} / \mathrm{Cit}$ and $\mathrm{Cho} / \mathrm{Cr}$ with different risk groups were $\mathrm{r}=0.32(p<0.05)$ and $\mathrm{r}=0.28(p<0.05)$, respectively. Figure 6 shows a boxplot representing the $\mathrm{ADC}$ and $(\mathrm{Cho}+\mathrm{Spm}+\mathrm{Cr}) / \mathrm{Cit}$ parameters for normal, low-risk, intermediate-risk, and high-risk tissues. Higher grade $\mathrm{TZ}$ cancer tissue was associated with lower $\mathrm{ADC}$ values (Fig. 6A) and higher $(\mathrm{Cho}+\mathrm{Spm}+\mathrm{Cr}) / \mathrm{Cit}$ ratios (Fig. 6B).

$\mathrm{K}^{\text {trans }}$ and ADC values were used to build RBF-SVM models between different risk groups (normal vs 


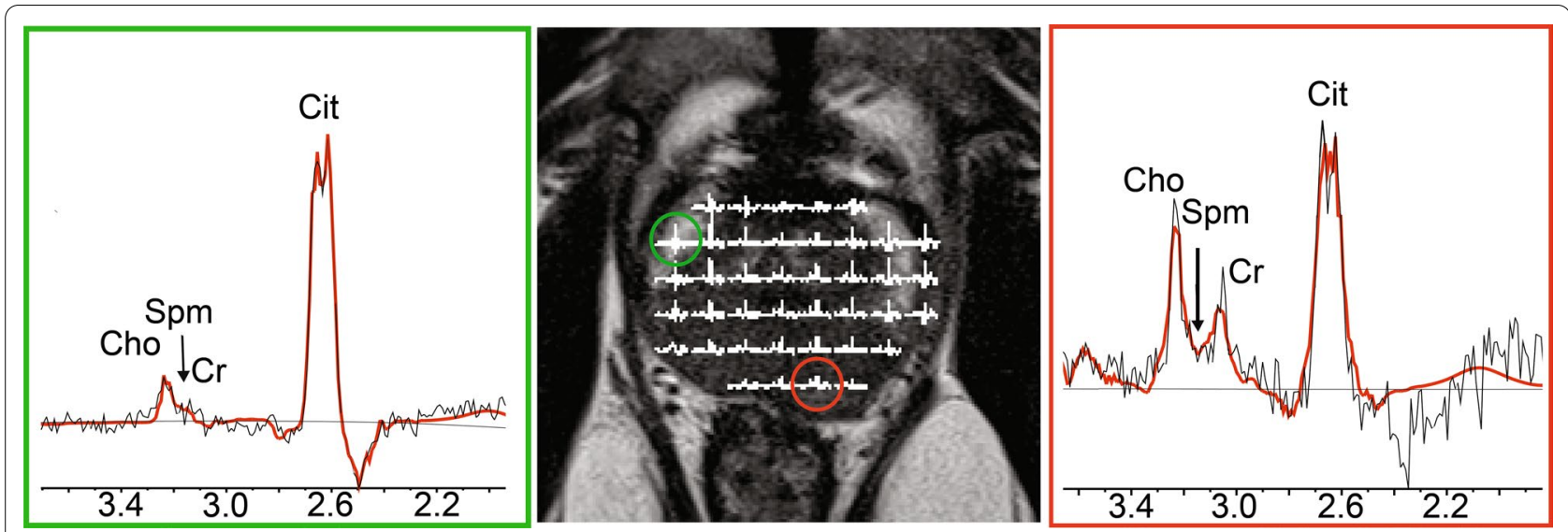

Fig. 4 T2Wl images with spectroscopy grid (middle panel) of a 72-year-old patient with an elevated serum PSA level of $14.5 \mathrm{ng} / \mathrm{ml}$, who was diagnosed with biopsy proven cancer lesions with Gleason $4+3$ in the left peripheral zone and a Gleason $3+3$ lesion in the left transition zone. The red circle identifies a voxel in cancer region (right hand side spectrum) and green circle identifies a voxel in normal tissue of the peripheral zone (left hand side spectrum)

Table 3 Mean \pm standard deviation of multiparametric magnetic resonance imaging (mp-MRI) parameters for discrimination of cancer and normal tissue in the central gland

\begin{tabular}{lccc}
\hline Parameters & Normal & $\begin{array}{l}\text { Cancer (low-risk, } \\
\text { intermediate-risk } \\
\text { and high-risk) }\end{array}$ & p-value \\
\hline $\mathrm{ADC}\left(10^{-6} \mathrm{~mm}^{2} / \mathrm{s}\right)$ & $1112.42 \pm 138.83$ & $871.10 \pm 149.61$ & $<0.01$ \\
$\mathrm{~K}^{\text {trans }}\left(\mathrm{min}^{-1}\right)$ & $0.14 \pm 0.08$ & $0.24 \pm 0.10$ & $<0.01$ \\
$\mathrm{~K}_{\mathrm{ep}}\left(\mathrm{min}^{-1}\right)$ & $0.65 \pm 0.38$ & $1.12 \pm 0.53$ & $<0.01$ \\
$\mathrm{iAUGC}(\mathrm{mmol} . \mathrm{min} / \mathrm{L})$ & $2.61 \pm 1.04$ & $3.15 \pm 1.31$ & $<0.05$ \\
$(\mathrm{Cho}+\mathrm{Spm}+\mathrm{Cr}) / \mathrm{Cit}$ & $0.23 \pm 0.09$ & $1.60 \pm 1.12$ & $<0.01$ \\
$\mathrm{Cho} / \mathrm{Cit}$ & $0.16 \pm 0.08$ & $0.82 \pm 0.38$ & $<0.01$ \\
$\mathrm{Cho} / \mathrm{Cr}$ & $1.28 \pm 0.68$ & $2.94 \pm 0.87$ & $<0.01$ \\
\hline
\end{tabular}

$A D C$ apparent diffusion coefficient, $K^{\text {trans }}$ the volume transfer constant, $K_{e p}$ the rate constant, iAUGC the area under gadolinium curve, Cho choline, Spm spermine, $\mathrm{Cr}$ creatine, Cit citrate

cancer, low-risk vs high-risk cancer, low-risk vs intermediate-risk cancer and intermediate-risk vs high-risk, $(p<0.05))$. Then, these models were extended by adding MRSI metabolite ratios to the $\mathrm{K}^{\text {trans }}$ and ADC values. The ROC curve of the final RBF-SVM model for each set of two risk groups was plotted and AUC's were determined to compare the diagnostic performance of each model (Fig. 7). The results demonstrate that metabolite ratios improve the AUC, sensitivity, specificity and accuracy for the discrimination of low-risk vs high-risk cancer and low-risk vs intermediate-risk cancer groups (right side of Fig. 7). For instance, the AUC in discriminating low-risk from high-risk cancer significantly increased by adding metabolite ratios from MRSI to the combination of ADC and $K^{\text {trans }}$ from 0.64 to $0.86(p<0.01)$. There was no significant difference between the performance of models for intermediaterisk vs high-risk cancer with and without MRSI metabolite ratios $(p>0.05)$.

\section{Discussion}

A combination of T2WI, DWI and DCE is the most commonly used mp-MRI set in routine clinical exams for the management of patients with localized PCa as defined by PI-RADS V2 guidelines [16]. Numerous studies evaluated a quantitative combination of these mpMRI methods to investigate their ability for the detection, localization, grading and staging of cancer in the prostate [16-18]. Most of these studies reported poor-tomoderate accuracy and reproducibility of mp-MRI for localization of $\mathrm{PCa}$ in $\mathrm{TZ}$ as compared to $\mathrm{PZ}[7,19-21]$. $\mathrm{TZ}$ of patients suspected of having $\mathrm{PCa}$ frequently contain regions of glandular $\mathrm{BPH}$ and stromal $\mathrm{BPH}$, next to other tissues such as (hypertrophic) anterior fibromuscular stroma [22]. In the diagnosis of TZ cancer, T2WI is usually emphasized more than DWI [23]. Because BPH can appear as hyperintense nodules and stromal $\mathrm{BPH}$ as hypointense nodules on T2WI, it remains challenging to differentiate $\mathrm{TZ}$ cancer from normal tissue using T2WI alone [24]. Combining T2WI and DWI generally improves $\mathrm{TZ}$ tumor detection and localization, although these results are variable $[21,25,26]$.

Our results indicate that the combination of T2WI and ADC's derived from DWI exams including high b-value $\left(1600 \mathrm{~s} / \mathrm{mm}^{2}\right)$, using RBF-SVM classification, improves the diagnostic accuracy of $\mathrm{TZ}$ cancer compared to T2WI alone. This result is similar to that reported previously for TZ [19, 27-29]. Some studies demonstrated better 


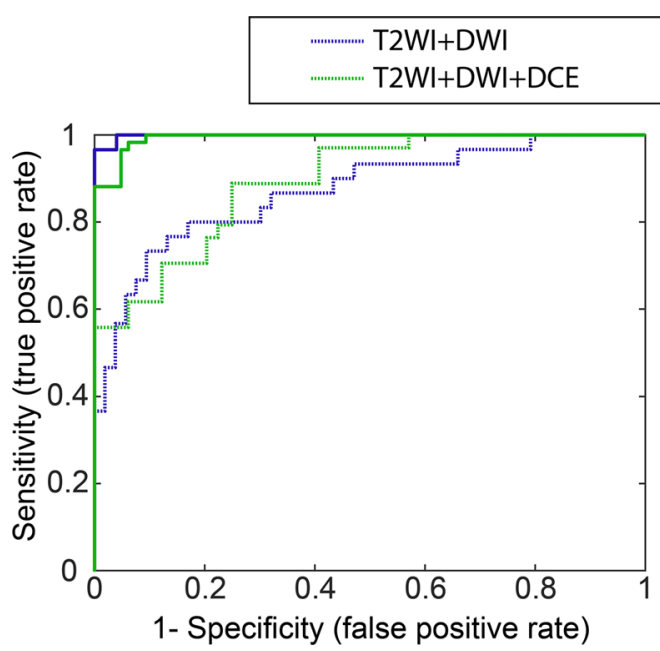

\section{$-\mathrm{T} 2 \mathrm{WI}+\mathrm{DW}+\mathrm{MRSI}$ \\ $-\mathrm{T} 2 \mathrm{WI}+\mathrm{DW}++\mathrm{DCE}+\mathrm{MRSI}$}

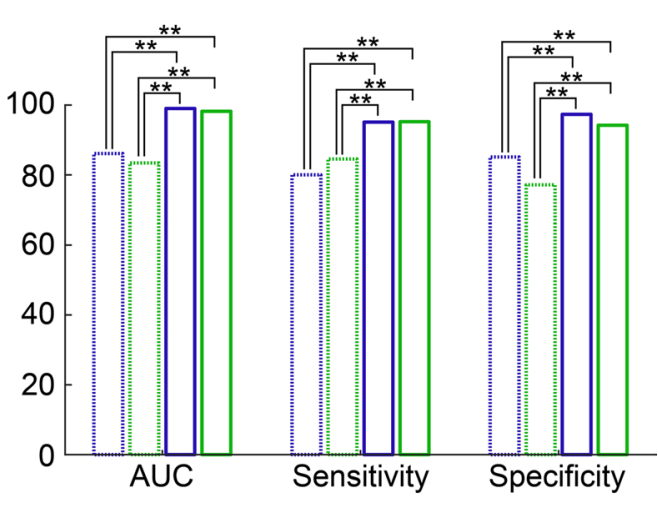

Fig. 5 Results of support vector machine analysis to discriminate cancer from normal tissue in the prostate transition zone. Left: ROC plot of combinations of multiparametric MRI values. T2WI + DWI is in blue and T2WI+DWI+DCE in green. Without MRSI is represented by a dashed-line and with MRSI by a solid-line. Only multiparametric MRI parameters with statistically significant differences between cancer and normal tissues $(p<0.05)$ were used for classification. Right: Bar charts of AUC values, sensitivity and specificity of the corresponding RBF-SVM models. McNemar test was used for pairwise comparison of sensitivity and specificities of models and Delong test was calculated for pairwise comparison of AUC of models. ${ }^{* *} p<0.01$

Table 4 Area under receiver operating characteristic curve (AUC) and diagnostic accuracy, sensitivity and specificity of detecting central cancers by T2WI (standardized T2WI signal intensity), DWI (ADC), DCE (Krans, $\mathrm{K}_{\mathrm{ep}}$ and $\left.\mathrm{AAUGC}\right)$ and MRSI ((Cho $\left.+\mathrm{Spm}+\mathrm{Cr}\right) / \mathrm{Cit}$, Cho/Cit and $\mathrm{Cho} / \mathrm{Cr}$ )

\begin{tabular}{lllll}
\hline Model & AUC (\%95 Cl) & Accuracy & Sensitivity & Specificity \\
\hline T2WI + DWI & $0.86(0.75-1.00)$ & $0.83(111 / 134)$ & $0.80(49 / 61)$ & $0.85(62 / 73)$ \\
T2WI + DWI + DCE & $0.83(0.70-1.00)$ & $0.80(107 / 134)$ & $0.84(51 / 61)$ & $0.77(56 / 73)$ \\
T2WI + DWI+ MRSI & $0.99(0.97-1.00)$ & $0.96(129 / 134)$ & $0.95(58 / 61)$ & $0.97(71 / 73)$ \\
T2WI + DWI+ DCE + MRSI & $0.98(0.95-1.00)$ & $0.95(127 / 134)$ & $0.95(58 / 61)$ & $0.94(69 / 73)$ \\
\hline
\end{tabular}

T2WIT2-weighted imaging, DWI diffusion weighted imaging, DCE dynamic contrast enhancement, $M R S /$ magnetic resonance spectroscopic imaging, $C l$ confidence intervals

results for $\mathrm{TZ}$ cancer detection by combining T2WI and higher b-value DWI (b-value $\left.=2000 \mathrm{~s} / \mathrm{mm}^{2}\right)[21,30]$.

For the combination of T2WI and DCE, some reports suggested a potential value for DCE parameters in the diagnosis of $\mathrm{TZ}$ cancer, but other studies failed to find any added benefit [20,31]. Our results showed no improvement in AUC, accuracy, and sensitivity in detecting TZ cancer by adding DCE to T2WI+DWI. This agrees with studies in which the detection of TZ cancer was not improved by adding DCE to T2WI [31, 32]. However, a quantitative assessment with linear regression indicated that washout in DCE may contribute to detection efficiency [17]. T2WI+DWI+DCE achieved higher sensitivity and lower accuracy and specificity than T2WI+DWI and T2WI+DCE models. This can be attributed to the marked hyper-vascularity of BPH nodules, which can show rapid enhancement as well as early washout. However, together these MRI methods are still limited for accurate detection and localization of PCa in TZ. An alternative approach to this is adding MRSI to mp-MRI exams, as prescribed in the original PI-RADS (V1) guidelines. Our results (Table 4 and Fig. 5) suggest that DCE can be replaced by MRSI using a GOIA-sLASER sequence to detect TZ PCa.

It is well established that MRSI can provide valuable metabolic information for the non-invasive assessment of PCa $[10,11]$. Several studies have concluded that adding MRSI to mp-MRI exams improves the diagnostic performance of detecting cancer in TZ [33, 34]. Also in a multicentre trial MRSI was shown to be able to discriminate between cancer and normal TZ tissues [35]. Most of the previous prostate MRSI studies employed PRESS with standard RF pulses at $\mathrm{TE}>100 \mathrm{~ms}$, representing a $\mathrm{T} 2$ penalty on all signals of interest. Due to the suboptimal 


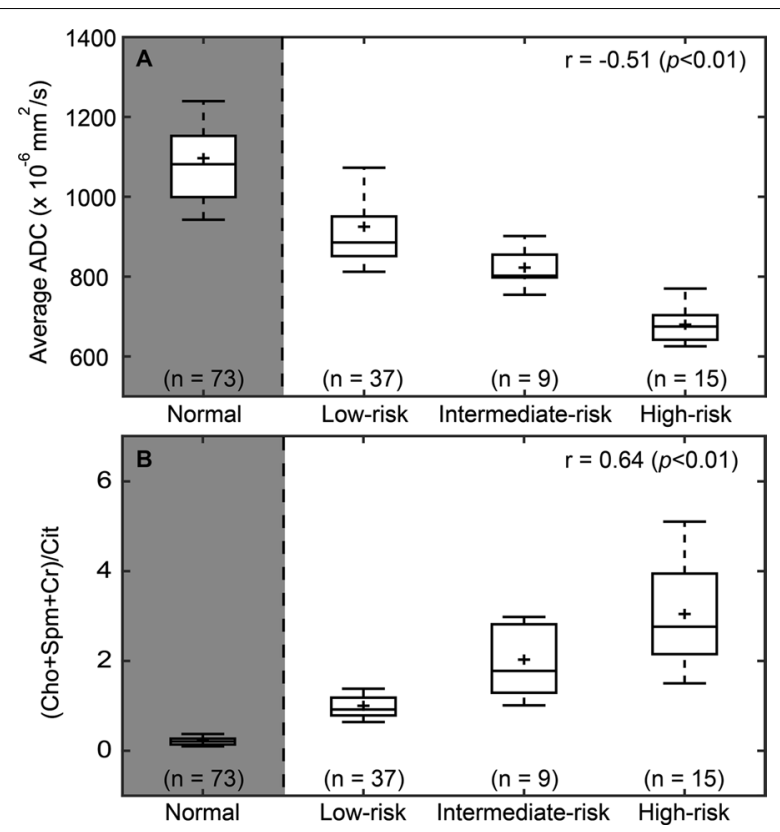

Fig. 6 Box plots of $\mathbf{A} A D C$ values and $\mathbf{B}$

(choline + spermine + creatine)/citrate $((\mathrm{Cho}+\mathrm{Spm}+\mathrm{Cr}) / \mathrm{Cit})$ values of low-risk, intermediate-risk and high-risk transition zone prostate cancer tissues. The correlations with low-risk, intermediate-risk and high-risk prostate cancer tissues were evaluated with Spearman correlation coefficients. MR parameters of ROls with normal transition zone tissue are shown for comparison (shaded) slice selection of standard RF pulses, causing unpredictable lipid signal contamination, and this $\mathrm{T} 2$ penalty, the added value of MRSI in mp-MRI of TZ was limited, in particularly when performed without using an endorectal coil [32].

The results of this study demonstrate that metabolic information derived from MRSI using GOIA-sLASER can accurately differentiate among low-risk and clinically significant $\mathrm{PCa}$ in $\mathrm{TZ}$. These results also indicate that adding MRSI data, acquired with a GOIA-sLASER sequence, to the routine clinical MRI exam can significantly improve accuracy. The useful role of quantitative MRSI parameters in combination with functional parameters in TZ tumor detection has been acknowledged in the literature [33, 36] Using an RBF-SVM model generated from T2WI, ADC and the metabolite ratios, we obtained high values for AUC, accuracy, sensitivity and specificity for diagnostic separation of cancer from normal. A similar performance was reported for a quantitative study in which TZ PCa was discriminated from normal tissue using an endorectal coil at a $\mathrm{TE}=85 \mathrm{~ms}$ and combining the Cho/Cr ratio from MRSI and ADC values [17].

In addition to the above, the correlation of mp-MRI parameters with low-risk $(\mathrm{GS}=3+3)$, intermediate-risk $(\mathrm{GS}=3+4)$ and high-risk $(\mathrm{GS} \geq 4+3)$ cancers was investigated. Quantitative T2WI and pharmacokinetic DCE parameters did not correlate with the GS risk groups except for a low correlation with $\mathrm{K}^{\text {trans }}$. This could be due to heterogeneity in tumor and normal tissue perfusion within TZ. The individual parameters $(\mathrm{Cho}+\mathrm{Spm}+\mathrm{Cr}) /$

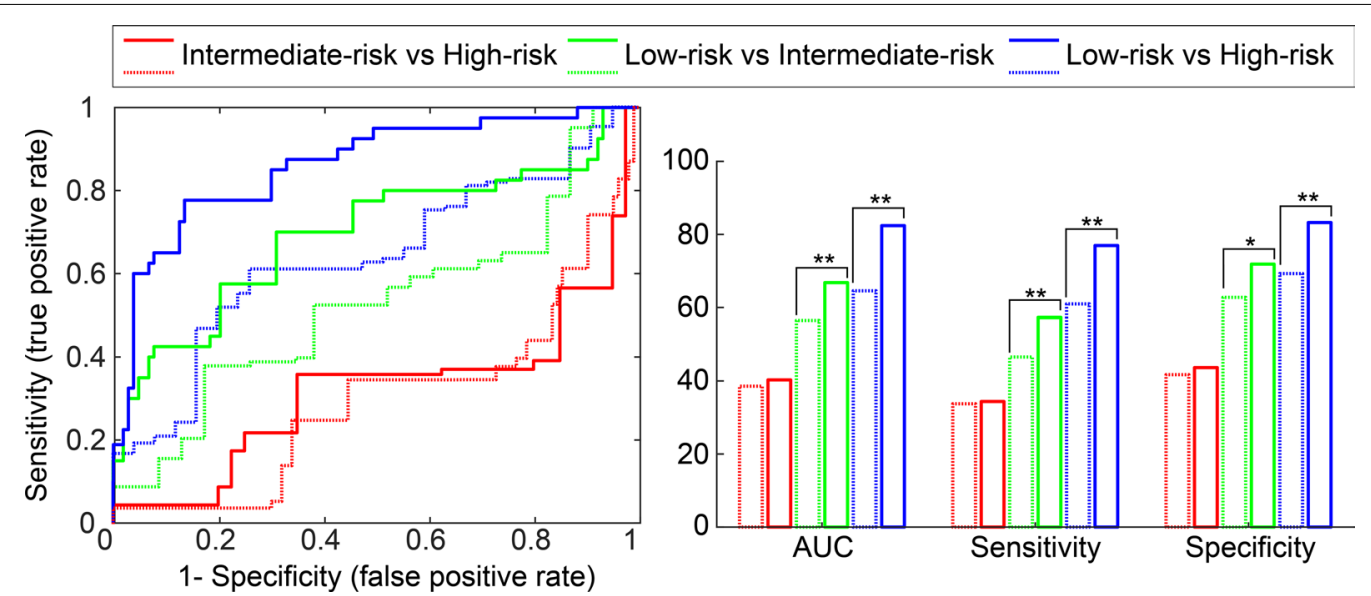

Fig. 7 Results of support vector machine analysis to separate tumor aggressiveness classes. Left side: ROC curves of the six RBF-SVM models for low-risk vs high-risk cancer, low-risk vs intermediate-risk cancer and intermediate-risk vs high-risk cancer. A leave-one-out cross-validation technique was used for the combined $A D C$ and $K^{\text {trans }}$ (dashed line) and all the combined ADC, $K^{\text {trans }}$ and metabolite ratios (solid line) with a significant difference between the two groups ( $p<0.05$ ). Right side: bar charts of AUC values, sensitivity and specificity of the corresponding RBF-SVM models. McNemar test was used for pairwise comparison of sensitivity and specificities of models and Delong test was calculated for pairwise comparison of AUC of models. ${ }^{* *} p<0.01$ and ${ }^{*} p<0.05$ 
Cit and ADC showed a moderate correlation with tumor aggressiveness. However, by combining $\mathrm{K}^{\text {trans }}, \mathrm{ADC}$ and metabolite ratios in an RBF-SVM model we found an AUC of 0.86 for the discrimination of low-risk from highrisk cancers.

The identification of low-risk vs high-risk cancers is of clinical importance as it may be used to avoid overtreatment of patients with $\mathrm{PCa}$. Comparable performances in the separation of low from high-risk cancer were obtained in studies of TZ using an endorectal coil and regression models involving ADC or DCE washout and metabolites from MRSI data [17, 37].

It is worth noting that 3D PRESS MRSI has been demonstrated to give reproducible results at $1.5 \mathrm{~T}$ with an endorectal coil in a test-retest setting [38]. In later publications it was demonstrated that 3D GOIA-sLASER MRSI is superior to 3D PRESS MRSI [12] and that the former can be applied to the prostate without using an endorectal coil producing reliable metabolic values [39]. One of the limitations of the current study is the relatively small number of intermediate and high grade $\mathrm{TZ}$ cancer patients for classification and prediction. Another limitation of this study is that the histopathological classification of lesions is based on a biopsy instead of on whole mount sections.

\section{Conclusion}

This study demonstrated that MRSI with a GOIA-sLASER sequence in combination with structural T2WI and DWI offers a non-invasive and reliable tool to assess cancer tissue in the central prostate gland. We found that DCE has limited value in TZ cancer detection and localization. Although the correlation between cancer aggressiveness and metabolic ratios or ADC values was moderate, the combination of these two enabled a good separation between low-risk and high-risk cancers in TZ.

\section{Abbreviations}

3D: Three dimensional; ADC: Apparent diffusion coefficient; AUC: Area under curve; BPH: Benign prostatic hyperplasia; Cho: Choline; Cit: Citrate; Cr: Creatine; CRLB: Cramer-Rao lower bound; CSDE: Chemical shift displacement error; DCE: Dynamic contrast enhancement; DRE: Digital rectal exam; DWI: Diffusionweighted imaging; FWHM: Full width at half maximum; GOIA: Gradient-modulated offset-independent adiabatic; GS: Gleason score; iAUGC: Area under gadolinium curve; $K^{\text {trans }}$ : Volume transfer constant; $K_{\mathrm{ep}}$ : Rate constant; Mp-MRI: Multiparametric MR imaging; MRSI: Magnetic resonance spectroscopic imaging; PCa: Prostate cancer; PSA: Prostate specific antigen; PI-RADS: Prostate imaging reporting and data system; PRESS: Point-resolved spectroscopy; PZ: Peripheral zone; RBF: Radial basis function; ROC: Receiver operator characteristic; ROI: Region of interest; sLASER: Semi-localized adiabatic selective refocusing pulse sequence; Spm: Spermine; SVM: Support vector machine; TRUS: Transrectal ultrasound; TZ: Transition zone; T2WI:T2-weighted imaging; VOI: Volume of interest.

\section{Acknowledgements}

Authors would especially like to acknowledge the contribution of the Clinical Research and Statistical Support unit in Hunter Medical Research Institute
(HMRI). We also would like to thank pathologists in Douglass Hanly Moir Pathology, Pathology North Hunter and Pathology of Maitland private Hospital for interpreting the biopsy tissues. The authors acknowledge the facilities and scientific and technical assistance of the National Imaging Facility, a National Collaborative Research Infrastructure Strategy (NCRIS) capability, at the HMRI Imaging Centre, University of Newcastle.

\section{Authors' contributions}

PG, SR and JS conducted study design. NG contributed to data collection, NG, SR, PG, JG and AH contributed to data analysis. PL and SS provided the segmentation. NG prepared the original draft and SR, PG, JS, JG, AH, CF reviewed and edited the final manuscript. All authors read and approved the final manuscript.

\section{Funding}

This work was funded by the Hunter Cancer Research Alliance (HCRA), Australia, Grant number: G1301098.

\section{Availability of data and materials}

The datasets generated during and/or analysed during the current study are available from the corresponding author on reasonable request.

\section{Declarations}

\section{Ethics approval and consent to participate}

The study protocol was approved by the local Human Research Ethics Committee, NSW, Australia. All patient signed a written consent form to participate in this study.

\section{Consent for publication}

A written and signed consent form was obtained from each patient for publication of their MR images.

\section{Competing interests}

The authors declare that they have no competing interests.

\section{Author details}

${ }^{1}$ School of Health Sciences, Faculty of Health and Medicine, University of Newcastle, Newcastle, NSW, Australia. ${ }^{2}$ School of Mathematical and Physical Sciences, University of Newcastle, Newcastle, NSW, Australia. ${ }^{3}$ Calvary Mater Newcastle, Radiation Oncology Department, Newcastle, NSW, Australia. ${ }^{4}$ MR Application Development, Siemens Shenzhen Magnetic Resonance Ltd., Shenzhen, China. ${ }^{5}$ Radiology Department, Calvary Mater Newcastle, Newcastle, NSW, Australia. ${ }^{6}$ Hunter Medical Research Institute (HMRI) Imaging Centre, New Lambton Heights, NSW, Australia. ${ }^{7}$ Department of Radiology and Nuclear Medicine, Radboud University Medical Center, Nijmegen, The Netherlands.

Received: 30 March 2021 Accepted: 10 July 2021

Published online: 19 July 2021

\section{References}

1. Bray F, Ferlay J, Soerjomataram I, Siegel RL, Torre LA, Jemal A. Global cancer statistics 2018: GLOBOCAN estimates of incidence and mortality worldwide for 36 cancers in 185 countries. CA Cancer J Clin. 2018:68:394-424.

2. McNeal JE, Redwine EA, Freiha FS, Stamey TA. Zonal distribution of prostatic adenocarcinoma. Correlation with histologic pattern and direction of spread. Am J Surg Pathol. 1988;2:897-906.

3. Augustin $H$, Erbersdobler $A$, Hammerer PG, Graefen M, Huland $H$. Prostate cancers in the transition zone: Part 2; clinical aspects. BJU Int. 2004;94:1226-9.

4. Oto A, Kayhan A, Jiang Y, Tretiakova M, Yang C, Antic T, et al. Prostate cancer: differentiation of central gland cancer from benign prostatic hyperplasia by using diffusion-weighted and dynamic contrast-enhanced MR imaging. Radiology. 2010;257:715-23.

5. Gordetsky JB, Thomas JV, Nix JW, Rais-Bahrami S. Higher prostate cancer grade groups are detected in patients undergoing multiparametric 
MRl-targeted biopsy compared with standard biopsy. Am J Surg Pathol. 2017:41:101-5.

6. Panebianco V, Giganti F, Kitzing YX, Cornud F, Campa R, De Rubeis G, et al. An update of pitfalls in prostate mpMRI: a practical approach through the lens of PI-RADS v. 2 guidelines. Insights Imaging. 2018;9:87-101.

7. Smith CP, Harmon SA, Barrett T, Bittencourt LK, Law YM, Shebel H, et al. Intra- and interreader reproducibility of PI-RADSv2: a multireader study. J Magn Reson Imaging. 2019;49:1694-703.

8. Bourne R, Panagiotaki E. Limitations and Prospects for Diffusion-Weighted MRI of the Prostate. Diags. 2016;6:21.

9. Verma S, Turkbey B, Muradyan N, Rajesh A, Cornud F, Haider MA, et al. Overview of dynamic contrast-enhanced MRI in prostate cancer diagnosis and management. Am J Roentgenol. 2012;198:1277-88.

10. Kobus T, Wright AJ, Scheenen TW, Heerschap A. Mapping of prostate cancer by 1 H MRSI. NMR Biomed. 2014;27:39-52.

11. Kurhanewicz J, Vigneron DB. Magnetic resonance spectroscopy of prostate cancer. eMagRes. 2016;1:923-44.

12. Steinseifer IK, van Asten JJ, Weiland E, Scheenen TW, Maas MC, Heerschap A. Improved volume selective 1 H MR spectroscopic imaging of the prostate with gradient offset independent adiabaticity pulses at 3 tesla. Magn Reson Med. 2015;74:915-24.

13. Gholizadeh N, Fuangrod T, Greer PB, Lau P, Ramadan S, Simpson J. An inter-centre statistical scale standardisation for quantitatively evaluating prostate tissue on T2-weighted MRI. Australas Phys Eng Sci Med. 2019;42:137-47.

14. Tofts PS, Brix G, Buckley DL, Evelhoch JL, Henderson E, Knopp MV, et al. Estimating kinetic parameters from dynamic contrast-enhanced T1-weighted MRI of a diffusable tracer: standardized quantities and symbols. J Magn Reson Imaging. 1999;10:223-32.

15. Gholizadeh N, Greer PB, Simpson J, Fu C, Al-iedani O, Lau P, et al. Supervised risk predictor of central gland lesions in prostate cancer using (1) $\mathrm{H}$ MR spectroscopic imaging with gradient offset-independent adiabaticity pulses. J Magn Reson Imaging. 2019;50:1926-36.

16. Ueno Y, Tamada T, Bist V, Reinhold C, Miyake H, Tanaka U, et al. Multiparametric magnetic resonance imaging: current role in prostate cancer management. Int J Urol. 2016;23:550-7.

17. Starobinets O, Simko JP, Kuchinsky K, Kornak J, Carroll PR, Greene KL, et al. Characterization and stratification of prostate lesions based on comprehensive multiparametric MRI using detailed whole-mount histopathology as a reference standard. NMR Biomed. 2017;30:e3796.

18. Padhani AR, Weinreb J, Rosenkrantz AB, Villeirs G, Turkbey B, Barentsz J. Prostate imaging-reporting and data system steering committee: PIRADS v2 status update and future directions. Eur Urol. 2019;75:385-96.

19. Hoeks CM, Vos EK, Bomers JG, Barentsz JO, de Kaa CA, Scheenen TW. Diffusion-weighted magnetic resonance imaging in the prostate transition zone: histopathological validation using magnetic resonance-guided biopsy specimens. Invest Radiol. 2013;48:693-701.

20. Futterer JJ, Heijmink SW, Scheenen TW, Veltman J, Huisman HJ, Vos P, et al. Prostate cancer localization with dynamic contrast-enhanced MR imaging and proton MR spectroscopic imaging. Radiology. 2006;241:449-58.

21. Rosenkrantz AB, Kim S, Campbell N, Gaing B, Deng FM, Taneja SS. Transition zone prostate cancer: revisiting the role of multiparametric MRI at 3 T. Am J Roentgenol. 2015;204:W266-72.

22. Gholizadeh N, Pundavela J, Nagarajan R, Dona A, Quadrelli S, Biswas T, et al. Nuclear magnetic resonance spectroscopy of human body fluids and in vivo magnetic resonance spectroscopy: potential role in the diagnosis and management of prostate cancer. Urol Oncol. 2020;38:150-73.

23. Barentsz J, de Rooij M, Villeirs G, Weinreb J. Prostate imaging-reporting and data system version 2 and the implementation of high-quality prostate magnetic resonance imaging. Eur Urol. 2017;72:189-91.

24. Hegde JV, Mulkern RV, Panych LP, Fennessy FM, Fedorov A, Maier SE, et al. Multiparametric MRI of prostate cancer: an update on state-of-the-art techniques and their performance in detecting and localizing prostate cancer. J Magn Reson Imaging. 2013;37:1035-54.

25. Verma S, Rajesh A, Morales H, Lemen L, Bills G, Delworth M, et al. Assessment of aggressiveness of prostate cancer: correlation of apparent diffusion coefficient with histologic grade after radical prostatectomy. Am J Roentgenol. 2011;196:374-81.

26. Tamada T, Sone T, Jo Y, Yamamoto A, Ito K. Diffusion-weighted MRI and its role in prostate cancer. NMR Biomed. 2014;27:25-38.

27. Akin O, Sala E, Moskowitz CS, Kuroiwa K, Ishill NM, Pucar D, et al. Transition zone prostate cancers: features, detection, localization, and staging at endorectal MR imaging. Radiol. 2006;239:784-92.

28. Delongchamps NB, Rouanne M, Flam T, Beuvon F, Liberatore M, Zerbib $\mathrm{M}$, et al. Multiparametric magnetic resonance imaging for the detection and localization of prostate cancer: combination of T2-weighted, dynamic contrast-enhanced and diffusion-weighted imaging. BJU Int. 2011:107:1411-8

29. Yoshizako T, Wada A, Hayashi T, Uchida K, Sumura M, Uchida N, et al. Usefulness of diffusion-weighted imaging and dynamic contrast-enhanced magnetic resonance imaging in the diagnosis of prostate transition-zone cancer. Acta Radiol. 2008;49:1207-13.

30. Woo S, Suh CH, Kim SY, Cho JY, Kim SH. Head-to-head comparison between high- and standard-b-value dwi for detecting prostate cancer: a systematic review and meta-analysis. Am J Roentgenol. 2017;210:91-100.

31. Hoeks CM, Hambrock T, Yakar D, van de Kaa CA, Feuth T, Witjes JA, et al. Transition zone prostate cancer: detection and localization with 3-T multiparametric MR imaging. Radiology. 2013;266:207-17.

32. Maas MC, Litjens GJ, Wright AJ, Attenberger UI, Haider MA, Helbich TH, et al. A single-arm, multicenter validation study of prostate cancer localization and aggressiveness with a quantitative multiparametric magnetic resonance imaging approach. Invest Radiol. 2019;54:437-47.

33. Kobus T, Vos PC, HambrockT, De Rooij M, Van de Kaa CA, Barentsz JO, et al. Prostate cancer aggressiveness: in vivo assessment of MR spectroscopy and diffusion-weighted imaging at 3 T. Radiol. 2012;265:457-67.

34. Turkbey B, Mani H, Shah V, Rastinehad AR, Bernardo M, Pohida T, et al. Multiparametric 3T prostate magnetic resonance imaging to detect cancer: histopathological correlation using prostatectomy specimens processed in customized magnetic resonance imaging based molds. J Urol. 2011;186:1818-24.

35. Scheenen TW, Fütterer J, Weiland E, Van Hecke P, Lemort M, Zechmann $C$, et al. Discriminating cancer from noncancer tissue in the prostate by 3-dimensional proton magnetic resonance spectroscopic imaging: a prospective multicenter validation study. Invest Radiol. 2011;46:25-33.

36. Riches SF, Payne GS, Morgan VA, Sandhu S, Fisher C, Germuska M, et al. MRI in the detection of prostate cancer: combined apparent diffusion coefficient, metabolite ratio, and vascular parameters. Am J Roentgenol. 2009:193:1583-91.

37. Vos EK, Kobus T, Litjens GJ, HambrockT, van de Kaa CA, Barentsz JO, et al. Multiparametric magnetic resonance imaging for discriminating lowgrade from high-grade prostate cancer. Invest Radiol. 2015;50:490-7.

38. Lagemaat MW, Zechmann CM, Fütterer JJ, Weiland E, Lu J, Villeirs GM, et al. Reproducibility of 3D $1 \mathrm{H}$ MR spectroscopic imaging of the prostate at 1.5 T. J Magn Reson Imaging. 2012;35:166-73.

39. Tayari N, Steinseifer IK, Selnæs KM, Bathen TF, Maas MC, Heerschap A. High-quality 3-dimensional $1 \mathrm{H}$ magnetic resonance spectroscopic imaging of the prostate without endorectal receive coil using a semi-LASER sequence. Invest Radiol. 2017;52:640-6.

\section{Publisher's Note}

Springer Nature remains neutral with regard to jurisdictional claims in published maps and institutional affiliations. 